Draft Version June 12, 2013

Preprint typeset using $\mathrm{LAT}_{\mathrm{E} X}$ style emulateapj v. 04/21/05

\title{
INTERMITTENT MILLISECOND X-RAY PULSATIONS FROM THE NEUTRON-STAR X-RAY TRANSIENT SAX J1748.9-2021 IN THE GLOBULAR CLUSTER NGC 6440
}

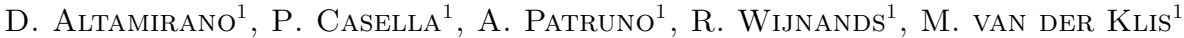 \\ Draft version June 12, 2013
}

\begin{abstract}
We report on intermittent X-ray pulsations with a frequency of $442.36 \mathrm{~Hz}$ from the neutron-star X-ray binary SAX J1748.9-2021 in the globular cluster NGC 6440. The pulsations were seen during both 2001 and 2005 outbursts of the source, but only intermittently, appearing and disappearing on timescales of hundreds of seconds. We find a suggestive relation between the occurrence of type-I $\mathrm{X}$-ray bursts and the appearance of the pulsations but the relation is not strict. This behavior is very similar to that of the intermittent accreting millisecond X-ray pulsar HETE J1900.1-2455. The reason for the intermittence of the pulsations remains unclear. However it is now evident that a strict division between pulsating and non-pulsating does not exist. By studying the Doppler shift of the pulsation frequency we determine an orbit with a period of $8.7 \mathrm{hrs}$ and an projected semi major axis of 0.39 lightsec. The companion star might be a main-sequence or a slightly evolved star with a mass of $\sim 1 M_{\odot}$. Therefore, SAX J1748.9-2021 has a longer period and may have a more massive companion star than all the other accreting millisecond X-ray pulsars except for Aql X-1.

Subject headings: binaries: general-pulsars: individual (SAX J1748.9-2021)-stars: neutron
\end{abstract}

\section{INTRODUCTION}

Accreting millisecond pulsars (AMPs, Alpar et al. 1982; Backer et al. 1982) are transient low mass X-ray binaries (LMXBs) that show X-ray pulsations during their outbursts. A total of nine AMPs out of 100 non-pulsating LMXBs have been found to date. The reason why only this small subgroup of binaries pulsates is still unknown. The first seven AMPs discovered showed persistent X-ray pulsations throughout the outbursts. Recently Kaaret et al. (2006) discovered the AMP HETE J1900.1-2455, which has remained active for more than 2 years $^{2}$ but showed pulsations only intermittently during the first $\sim 2$ months of activity (Galloway et al. 2007). From the transient source Aql X-1 pulsations were detected (Casella et al. 2007) only for $\sim 150$ sec out of the $\sim 1.3$ Msec the source has (so far) been observed with the Rossi X-ray Time Explorer (RXTE).

Gavriil et al. (2006, 2007) recently reported on the detection of $\sim 442.3 \mathrm{~Hz}$ pulsations in an observation of the 2005 outburst of a transient source in the globular cluster (GC) NGC 6440. The pulsations followed a flux decay observed at the beginning of the observation and were reminiscent of those observed during superbursts; however, as Gavriil et al. (2007) suggest, they could also be a detection from a new intermittent accreting millisecond pulsar. Kaaret et al. (2003) report a $409.7 \mathrm{~Hz}$ burst oscillation in an X-ray transient (SAX J1748.9-2021) located also in NGC 6440 and this GC harbors at least $24 \mathrm{X}$ ray sources (Pooley et al. 2002), so Gavriil et al. (2007) concluded that the burst oscillations and the pulsations were probably coming from different X-ray transients in the same GC.

\footnotetext{
${ }^{1}$ Email: diego@science.uva.nl ; Astronomical Institute, "Anton Pannekoek", University of Amsterdam, and Center for High Energy Astrophysics, Kruislaan 403, 1098 SJ Amsterdam, The Netherlands.

${ }^{2}$ At the time of submitting this letter, the source is still active.
}

The exact formation mechanisms behind the pulsations of these three sources remains unknown. The existence of intermittent pulsations with a small duty cycle implies that many other apparently non-pulsating LMXBs might be pulsating, bridging the gap between the small number of AMPs and the large group of non-pulsating LMXBs.

We are performing a detailed analysis of all RXTE archival data of neutron-star LMXBs to search for transient pulsations in their X-ray flux (see also Casella et al. 2007). In this Letter we present the results of our search on the three X-ray outbursts observed from the globular cluster NGC 6440.

\section{THE NEUTRON-STAR TRANSIENT SAX J1748.9-2021 IN NGC 6440}

NGC 6440 is a GC at $8.5 \pm 0.4 \mathrm{kpc}$ Ortolani et al. 1994). Bright X-ray outbursts from a LMXB were reported in 1971, 1998, 2001 and 2005 (Markert et al. 1975; in 't Zand et al. 1999; Verbunt et al. 2000; in't Zand et al. 2001; Markwardt \& Swank 2005). in't Zand et al. (2001) from X-ray and optical observations concluded that the 1998 and 2001 outbursts were from the same object, which they designated SAX J1748.9-2021.

\section{OBSERVATIONS, DATA ANALYSIS AND RESULTS}

We used data from the RXTE Proportional Counter Array (PCA, for instrument information see Jahoda et al. 2006). Up to July, 2007, there were 27 pointed observations of SAX J1748.9-2021, each covering 1 to 5 consecutive $90-\mathrm{min}$ satellite orbits. Usually, an orbit contains between 1 and $5 \mathrm{ksec}$ of useful data separated by 1-4 ksec data gaps due to Earth occultations and South Atlantic Anomaly passages. Adopting a source position $\left(\alpha=17^{h} 48^{m} 52^{s} .163\right.$, $\delta=-20^{\circ} 21^{\prime} 32^{\prime \prime}$.40; J2000 Pooley et al. 2002) we converted the $2-60 \mathrm{keV}$ photon arrival times to the Solar System barycenter with the FTOOL faxbary, which uses the JPL DE-200 ephemeris along with the spacecraft 



FIG. 1. - Intensity $(2.0-16.0 \mathrm{keV})$ normalized by Crab vs. time of the three outbursts. Gray symbols show the 16 -sec averaged intensity during the pointed PCA observations. The continuous line shows the ASM light curve. Black marks at the top mark the times of type-I $\mathrm{X}$-ray bursts. Black marks at the bottom mark the times when we detect significant pulsations. Years of the outburst is shown in each panel.

TABLE 1

TIMING PARAMETERS FOR NGC 6440

\begin{tabular}{|c|c|}
\hline Parameter & Value \\
\hline Orbital period, $\mathrm{P}_{\text {orb }}(\mathrm{hr})$ & $8.764(6) \mathrm{hr}$ \\
\hline Projected semi major axis, $a_{x} \operatorname{sini}$ (lightsec.) & $0.39(1)$ \\
\hline Epoch of $0^{\circ}$ mean longitude ${ }^{1}, T_{0}$ (MJD/TDB) & $52190.047(4)$ \\
\hline Eccentricity, e .................. & $<0.001$ \\
\hline Spin frequency $\nu_{0}(\mathrm{~Hz})$ & $442.361(1)$ \\
\hline Pulsar mass function, $f_{x}\left(\times 10^{-4} M_{\odot}\right) \ldots \ldots \ldots \ldots \ldots \ldots$ & $\simeq 4.8$ \\
\hline Minimum companion mass, $M_{c}\left(M_{\odot}\right) \ldots \ldots \ldots \ldots \ldots \ldots$ & $\gtrsim 0.1$ \\
\hline
\end{tabular}
to the ascending node of the orbit.

ephemeris and fine clock corrections to provide an absolute timing accuracy of $5-8 \mu \mathrm{s}$ (Rots et al. 2004).

We performed a Fourier timing analysis using the high-time resolution data collected in the Event (E_125us_64M_0_1s) and the Good Xenon modes. Power spectra were constructed using data segments of 128 , 256 and 512 seconds and with a Nyquist frequency of $4096 \mathrm{~Hz}$. No background or dead-time corrections were made prior to the calculation of the power spectra, but all reported rms amplitudes are background corrected; deadtime corrections are negligible.

\subsection{Colors, light curves and states}

From the Standard 2 data (Jahoda et al. 2006), we calculated colors and intensities with a time resolution of 16 seconds and normalized by Crab (e.g Altamirano et al. 2007). The PCA observations sample three different outbursts (see Fig. 1). The color-color diagrams show a pattern (not plotted) typical for atoll sources. The power spectral fits confirm the identification of these states (see also Kaaret et al. 2003). We looked for $\mathrm{kHz}$ QPOs, but found none.

No thermonuclear bursts were detected in the first outburst, sixteen during the second (Kaaret et al. 2003; Galloway et al. 2006) and four during the third one. We searched for burst oscillations during all bursts in the 15$4000 \mathrm{~Hz}$ frequency range but found none. Kaaret et al. $(2003)$ reported $\mathrm{a} \sim 4.4 \sigma$ burst oscillation at $\sim 409.7 \mathrm{~Hz}$. We find these authors underestimated the number of trials by a factor of at least 180, as their estimate did not take into account the number of X-ray bursts analyzed and the fact that a sliding window was used to find the maximum power. Moreover, we also found that the distribution of powers is not exponential as these authors assumed. Taking into account these effects we estimate
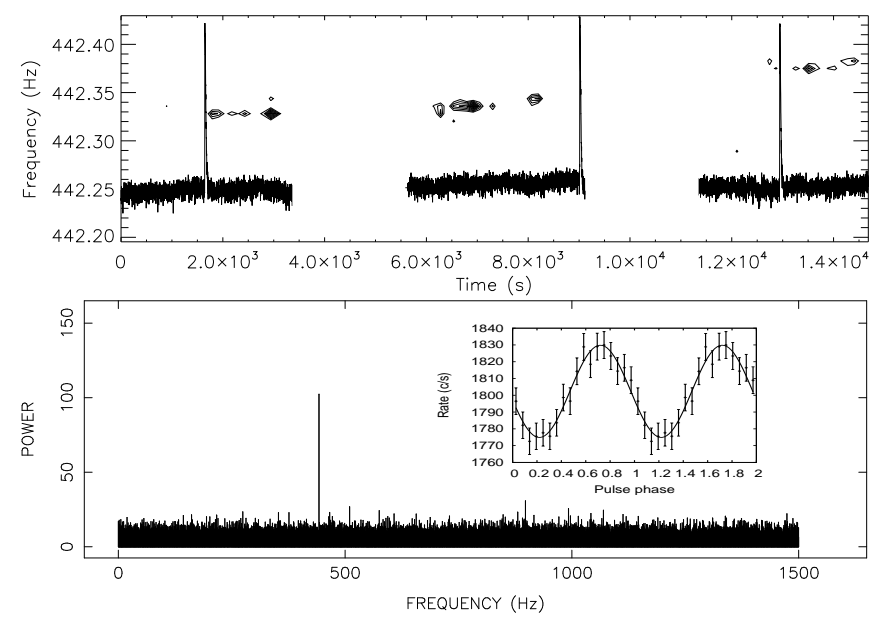

FIG. 2.- Top: Dynamical power spectrum of observation 6003502-03-00 showing intermittent pulsations (contours). In the light curve (line) three X-ray bursts are seen. The pulse frequency drifts due to orbital Doppler modulation. The lowest contour plotted corresponds to Leahy power 13 and the highest to 55 . The contours were generated from power spectra for non-overlapping $128 \mathrm{sec}$ intervals of data. Bottom: Leahy normalized (Leahy et al. 1983) power spectrum of $512 \mathrm{sec}$ of data centered $\sim 7 \mathrm{ksec}$ after the start of this observation. Maximum Leahy power is 102, corresponding to a single-trial probability of $\sim 7 \cdot 10^{-23}$ given Poissonian statistics in the photon arrival times (van der Klis 1995). Inset: The 2-60 $\mathrm{keV}$ light curve folded at the 2.26-ms period. Two cycles are plotted for clarity. The pulse profile is sinusoidal, with a $95 \%$ upper limit of $0.4 \%$ (rms) on the amplitude of the second harmonic.

the significance for their detection to be $\lesssim 2.5 \sigma$.

\subsection{Pulsations}

We inspected each power spectrum for significant features. We found several, at frequencies $\sim 442.3 \mathrm{~Hz}$ in 7 observations: 60035-02-02-04/05/06, 60035-02-0300/02/03 during the second outburst and 91050-03-07-00 during the third outburst (see also Gavriil et al. 2007, for a detailed analysis of this observation). in't Zand et al. (2001) concluded that the 1998 and 2001 outbursts from the LMXB in NGC 6440 were from the same source (Section 2). Since pulsations are detected in both the 2001 and 2005 outbursts, we can now conclude that these two outbursts are also from the same source. Hence, all outbursts observed from NGC 6440 over the last decade are from SAX J1748.9-2021.

The pulsations are detected intermittently, appearing and disappearing on time scales of hundreds of seconds. The appearance of pulsations seems to be related to the 


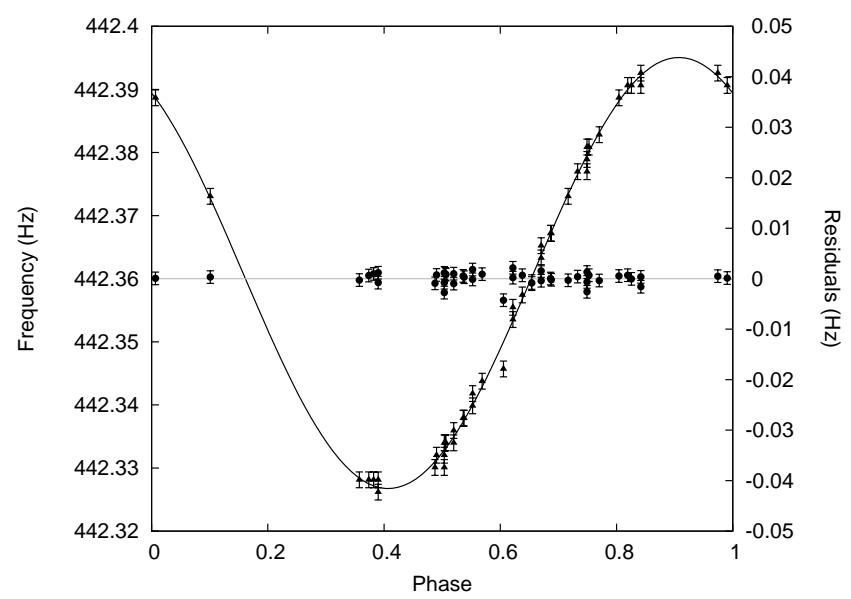

FIG. 3.- Pulse frequency as a function of orbital phase. The plot has been obtained by folding all data between the first and last pulse detection in 2001. Pulsations were detected during 6 of the 18 orbital cycles covered. Drawn curve is the best-fit orbital model, measured frequencies and post-fit residuals are shown. The residuals' r.m.s. is $1.2 \times 10^{-3} \mathrm{~Hz}$.

occurrence of type-I X-ray bursts, but the relation is not strict. The first two bursts were observed in an observation on October $8^{\text {th }} 2001$; the first pulsations a day later. During the third outburst we detect four bursts; pulsations were only detected after the third one. We also detected pulsations with no preceding burst. The structure of our data does not allow us to tell if pulsations and/or other bursts occurred during data gaps. Figure 2 (top) illustrates the relation between pulsations and bursts. The amplitude of the pulsations varies strongly between $\sim 2 \%$ and (often) undetectable $(0.3 \%$ rms amplitude upper limit at the $95 \%$ confidence level). Pulsations are seen right after the occurrence of the first and the third burst, but in the middle of the data pulsations are present without the detection of a preceding burst (although a burst could have happened just before the start of this data segment). In Figure 2 (bottom) we show a power spectrum and corresponding $2-60 \mathrm{keV}$ pulse profile (inset). In these data the pulsation is relatively hard; the rms amplitude increases with energy from $\sim 1 \%$ at $3 \mathrm{keV}$ to $\sim 3 \%$ at $13 \mathrm{keV}$.

The $2-10 \mathrm{keV}$ luminosity during the observations in which we detected pulsations was between 3 and $4 \times$ $10^{37} \mathrm{ergs} \mathrm{s}^{-1}$ (assuming a fixed $N_{H}=8.2 \times 10^{21} \mathrm{~cm}^{-2}$; in't Zand et al. 2001). Other observations at similar flux and those at higher ( up to $\simeq 5 \times 10^{37} \mathrm{ergs} \mathrm{s}^{-1}$ in observation 91050-03-06-00) and lower fluxes do not show pulsations (see Fig. 1). From 16, 32, 64 and 128 sec average colors we found no significant changes in the energy spectra correlated with the pulse-amplitude variations.

We studied the pulse frequency drifts using power spectra of 128, 256 and 512 sec data and find a clear 8.7 hours sinusoidal modulation which we interpret as due to Doppler shifts by binary orbital motion with that period. In order to obtain an orbital solution, we performed a $\chi^{2}$ scan on the orbital parameters using the method described by Kirsch et al. (2004) and Papitto et al. (2005). Our best estimates are listed in Table 1. The combination of data gaps and intermittency of the pulsations yielded aliases, which are taken into account by the reported errors. In Figure 3 we plot the pulse frequency as a function of orbital phase.

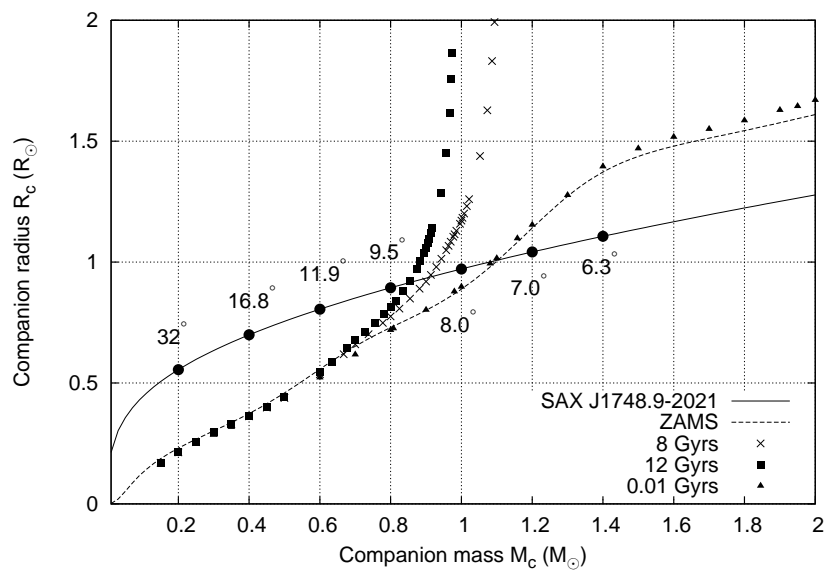

FIG. 4.- Mass-radius relationship for a Roche lobe-filling companion (continuous line), isochrones of $0.01,8$ and 12 Gyrs with solar metallicity (triangles, crosses and squares, respectively ; Girardi et al. 2000) and theoretical Zero-age main sequence (ZAMS, dashed line; Tout et al. 1996). Black circles mark the inclination of the system as estimated by the mass function of this system.

\section{DISCUSSION}

We have discovered intermittent pulsations from the neutron-star LMXB SAX J1748.9-2021. Pulsations appear and disappear on time scales of hundreds of seconds. Although we find a suggestive relation between the appearance of the pulsations and the occurrence of type-I X-ray bursts (the pulsations appearing after a burst), the relation is not strict. We find bursts with no subsequent pulsations and pulsations with no preceding burst (although a burst could have occurred in the preceding data gaps). From the Doppler shifts on the pulsations we determine that the system is in a near-circular orbit with period of 8.7 hours and projected radius of 0.39 lightsec.

The stability of the pulsations (after correcting for the binary orbit) strongly suggests that the pulsation frequency reflects the neutron star spin frequency and that SAX J1748.9-2021 is an accreting millisecond X-ray pulsar. The characteristics of the pulsations are reminiscent of the those found in HETE J1900.1-2455: in both sources the pulsations were only intermittently detected and a possible relation between burst occurrence and pulse amplitude exists. However, there are differences: in HETE J1900.1-2455 the pulsations were only seen during the first two months of the outburst and their amplitude decreased steadily on timescales of days after the bursts which might have caused them to reappear (Galloway et al.2007). In SAX J1748.9-2021 we find the pulsations in the middle of the 2001 and 2005 outbursts and not in the beginning. Furthermore, the amplitude of the pulsations behaves erratically, switching between detection and non-detection on time scales of hundreds of seconds. Despite these differences, the behavior of the pulsations in both sources is so similar that we consider it likely that the same mechanism causes the intermittency of the pulsations in both.

A related system might be Aql X-1 in which a shortlived ( $\sim 150 \mathrm{~s}$ ) and very rare (duty cycle of $0.03 \%$ ) episode of strong pulsations at the neutron-star spin frequency has been detected (Casella et al. 2007). In this source, no X-rays bursts were seen in the $\sim 1400 \mathrm{~s}$ before the pulsations, making it unlikely that they were triggered 
by a burst. It is unclear if the pulsations in Aql X-1 were accretion-driven or due to unusual nuclear burning episodes; the same applies to SAX J1748.9-2021. The extreme rarity of the pulsations in Aql X-1 could indicate that the mechanism behind them is different from that responsible for the pulsations in HETE J1900.12455 and SAX J1748.9-2021. Nevertheless, irrespective of the mechanisms behind the pulsations in these three sources, it is clear that a strict division between pulsating and non-pulsating sources cannot be made anymore. It is possible that all sources pulsate occasionally although the recurrence times could be very long.

Assuming a constant dipolar magnetic field, following Psaltis \& Chakrabarty (1999) (i.e., assuming a geometrically thin disk and neglecting inner disk wind mass loss, radiation drag and GR effects) we estimate the magnetic field to be $B \gtrsim 1.3 \times 10^{8}$ Gauss. This assumes a $10 \mathrm{~km}$ radius $1.4 M_{\odot}$ neutron star and $\dot{M}_{\max }$, the highest accretion rate at which pulses are detected, of 0.28 of the Eddington critical value as derived from the luminosity observed at the time using a bolometric flux correction of 1.4 (Migliari \& Fender 2006). In the standard magnetic channeling scenario, the question remains of what causes the large variations in pulse amplitude.

Comparisons between HETE J1900.1-2455, SAX J1748.9-2021 and the other 7 AMSPs can provide clues to understand the pulse-strength variations. In SAX J1748.9-2021 and Aql X-1 the time scales on which the pulse amplitude can fluctuate are as short as $\sim 10^{2} \mathrm{~s}$, too short for the properties of the neutron star core to change (Galloway et al. 2007). So, these changes must originate in the disk or the outer layers of the neutron star envelope. Galloway et al. (2007) suggested (for HETE J1900.1-2455) that the accumulation of matter on the surface burying the magnetic field (Cumming et al. 2001) plays a role. Our results show that this mechanism probably cannot work for SAX J1748.9-2021, as the pulsations are not seen in the beginning of the outbursts, but instead $\sim 3$ weeks and $\sim 5$ weeks after the start of the 2001 and 2005 outbursts, respectively, so after a considerable amount of matter has already accreted. Interestingly, we observe pulsations only around a mass accretion rate of $\simeq 2 \times 10^{-16} M_{\odot} / \mathrm{sec}$ as inferred from the X-ray luminosity, not above or below, indicating that instantaneous mass accretion rate rather than total accreted mass is the important quantity.

In both HETE J1900.1-2455 and SAX J1748.9-2021 the pulsations seem to appear together with bursts although the exact connection is complex. This suggests that surface processes may affect the magnetic field.
Hydrodynamic flows in the surface layer of the neutron star may screen the magnetic field (see review by Bhattacharva 2002, and references within); perhaps violent processes like bursts temporarily affect such flows, diminishing screening and enhancing the channeling.

Alternatively, variations in a scattering or screening medium may cause the pulse amplitude modulation (see e.g. discussions in Psaltis \& Chakrabarty (1999), Titarchuk et al. (2002), Göğüs et al. 2007, Titarchuk et al. 2007, Casella et al. 2007 and references within). For our results, the properties of such a medium should change on timescales of hundreds of seconds; note that we did not detect spectral changes associated with pulse strength modulation.

With an orbital period of $\sim 8.7$ hours, this binary system is clearly not an ultra-compact binary as usually found in globular clusters and in fact, SAX J1748.92021 is the AMSP with the longest orbital period after Aql X-1, which has an orbital period of $\sim$ 19 hrs (Chevalier \& Ilovaisky 1991; Welsh et al. 2000). The mass-radius relation for a low-mass Roche lobefilling companion in a binary (Eggleton 1983) is $R_{c}=$ $0.24 M_{N S}^{1 / 3} q^{2 / 3}(1+q)^{1 / 3} P_{h r}^{2 / 3} /\left(0.6 q^{2 / 3}+\log \left(1+q^{1 / 3}\right)\right)$, with $P_{h r}$ the orbital period in hours, $M_{N S}$ the mass of the neutron star, $R_{c}$ radius of the companion and $q=M_{c} / M_{N S}$, the mass ratio. Given the mass function and the orbital period and assuming a $1.4 M_{\odot}$ neutron star, we plot in Figure 4 the mass-radius relationship for the companion star. Given that the age of the Globular Cluster NGC 6440 is $10 \pm 2$ Gyrs (Santos \& Piatti 2004) and its metallicity is approximately solar (Ortolani et al. 1994), in Figure 4 we also plot the isochrones for stars with ages of 8 and 12 Gyrs and solar metallicity. Stars with a $M_{c}<0.85 \mathrm{M}_{\odot}$ cannot fill the Roche lobe while stars with $M_{c}>0.95 \mathrm{M}_{\odot}$ would have a radius exceeding the Roche lobe. This would imply a donor star mass of $0.90 \pm 0.05 M_{\odot}$. However, for masses of $0.95-1.1 \mathrm{M}_{\odot}$, stars have evolved off the main sequence so binary mass transfer can have affected the radius of the donor star, which means we cannot firmly exclude masses of 0.95 $1.1 \mathrm{M}_{\odot}$. Therefore a more conservative mass range for the donor star is $0.85-1.1 \mathrm{M}_{\odot}$. Intriguingly, this requires the inclination to be about $9^{\circ}$, which has a $\lesssim 1 \%$ a priori probability for an isotropic sample of binary inclinations. Of course, this estimate is assuming that SAX J1748.92021 is in a primordial binary. If a different evolutionary path took place (e.g. dynamical interactions), the mass of the companion might be much smaller (see e.g. van Zyl et al. 2004).

\section{REFERENCES}

Alpar M.A., Cheng A.F., et al., 1982, Nature, 300, 728

Altamirano D., van der Klis M., et al., 2007, Submitted to ApJ

Backer D.C., Kulkarni S.R., et al., 1982, Nature, 300, 615

Bhattacharya D., Jun. 2002, Journal of Astrophysics and Astronomy, 23, 67

Casella P., Altamirano D., et al., 2007, ApJL accepted

Chevalier C., Ilovaisky S.A., Nov. 1991, A\&A, 251, L11

Cumming A., Zweibel E., Bildsten L., 2001, ApJ, 557, 958

Eggleton P.P., 1983, ApJ, 268, 368

Galloway D.K., Muno M.P., et al., 2006, ArXiv e-prints

Galloway D.K., Morgan E.H., et al., 2007, ApJ, 654, L73
Gavriil F.P., Strohmayer T.E., et al., Sep. 2006, In: Bulletin of the American Astronomical Society, vol. 38 of Bulletin of the American Astronomical Society, 336-+

Gavriil F.P., Strohmayer T.E., et al., Nov. 2007, ApJ, 669, L29

Girardi L., Bressan A., et al., Feb. 2000, A\&AS, 141, 371

Göğüs E., Alpar M.A., Gilfanov M., 2007, ApJ, 659, 580

in 't Zand J.J.M., Verbunt F., et al., 1999, A\&A, 345, 100

in't Zand J.J.M., van Kerkwijk M.H., et al., 2001, ApJ, 563, L41

Jahoda K., Markwardt C.B., et al., 2006, ApJS, 163, 401

Kaaret P., Zand J.J.M.i., et al., 2003, ApJ, 598, 481

Kaaret P., Morgan E.H., et al., 2006, ApJ, 638, 963

Kirsch M.G.F., Mukerjee K., et al., Aug. 2004, A\&A, 423, L9

Leahy D.A., Darbro W., et al., 1983, ApJ, 266, 160 
Markert T.H., Backman D.E., et al., 1975, Nature, 257, 32

Markwardt C.B., Swank J.H., 2005, The Astronomer's Telegram, 495,1

Migliari S., Fender R.P., 2006, MNRAS, 366, 79

Ortolani S., Barbuy B., Bica E., 1994, A\&AS, 108, 653

Papitto A., Menna M.T., et al., Mar. 2005, ApJ, 621, L113

Pooley D., Lewin W.H.G., et al., 2002, ApJ, 573, 184

Psaltis D., Chakrabarty D., 1999, ApJ, 521, 332

Rots A.H., Jahoda K., Lyne A.G., 2004, ApJ, 605, L129

Santos J.F.C. Jr., Piatti A.E., 2004, A\&A, 428, 79

Titarchuk L., Cui W., Wood K., 2002, ApJ, 576, L49

Titarchuk L., Kuznetsov S., Shaposhnikov N., 2007, ArXiv e-prints, 706
Tout C.A., Pols O.R., et al., 1996, MNRAS, 281, 257

van der Klis M., 1995, Proceedings of the NATO Advanced Study Institute on the Lives of the Neutron Stars, held in Kemer, Turkey, August 19-September 12, 1993. Editor(s), M. A. Alpar,

U. Kiziloglu, J. van Paradijs; Publisher, Kluwer Academic, Dordrecht, The Netherlands, Boston, Massachusetts, 301 van Zyl L., Charles P.A., et al., May 2004, MNRAS, 350, 649 Verbunt F., van Kerkwijk M.H., et al., 2000, A\&A, 359, 960 Welsh W.F., Robinson E.L., Young P., Aug. 2000, AJ, 120, 943 